\title{
A single-case design investigation for measuring the efficacy of Gestalt therapy to treat depression in older adults with demen- tia in Italy and in Mexico: a research protocol
}

\author{
Alessandra Merizzi ${ }^{1 *}$, Rosanna Biasi ${ }^{2}$, José Fernando Álvarez Zamudio ${ }^{3}$, Margherita Spagnuolo-Lobb ${ }^{2}$ and Sara \\ Santini ${ }^{1}$ \\ 1 Centre for Socio-Economic Research on Aging, IRCCS INRCA-National Institute of Health and Science on \\ Aging, Via Santa Margherita 5, 60124 Ancona, Italy; a.merizzi@inrca.it; s.santini2@inrca.it \\ 2 Istituto di Gestalt HCC Human Communication Centre Italy, Via S. Sebastiano 38, 96100 Siracusa, Italy; \\ rosanna.biasi@gestalt.it; margherita.spagnuolo@gestalt.it \\ 3 Instituto Humanista de Psicoterapia Gestalt (IHPG), Africa 6, La Concepción, Coyoacán, 04020 Mexico City, \\ Mexico; fernandoalvarezzamudio@gmail.com \\ * Correspondence: a.merizzi@inrca.it
}

\begin{abstract}
Psychotherapy is one of the evidence-based clinical interventions for the treatment of depression in older adults with dementia. Randomized Controlled Trials are often the first methodological choice to gain evidence, yet they are not applicable to a wide range of humanistic psychotherapies. Amongst all, the efficacy of the Gestalt therapy (GT) is under-investigated. The purpose of this paper is to present a research protocol aiming to assess the effects of a GT-based intervention on people with dementia (PWD) and the indirect influence on their family carers. The study implements the Single-Case Experimental Design with Time-Series Analysis that will be carried out in Italy and Mexico. Ten people in each country, who received a diagnosis of dementia and present depressive symptoms, will be recruited. Eight or more GT sessions will be provided whose fidelity will be assessed by the GT Fidelity Scale. Quantitative outcome measures are foreseen for monitoring participants' depression, anxiety, quality of life, carers' burden, and the caregiving dyad mutuality, at baseline and follow-up. The advantages and limitations of the research design are considered. If GT will result effective in the treatment of depression in PWD, it could enrich the range of evidence-based interventions provided by healthcare services.
\end{abstract}

Keywords: Gestalt therapy; dementia; depression; single-case experimental design; psychosocial interventions

\section{Introduction}

Dementia is a global epidemic: it is estimated that there are over 55 million people living with dementia worldwide, whose number is expected to almost triple by 2050 . Most of this increase can be attributed to population ageing happening at an unparalleled fast rate in low- and, particularly, middle-income countries [1].

Dementia, also called Major Neurocognitive Disorder, is an umbrella term that describes a set of symptoms of cognitive, psychological and behavioral nature, that compromises the daily functioning of a person, the underlying cause of which may be one or more neurocognitive diseases, Alzheimer's Disease (AD) being the most common. Dementia can affect people at any age, but its prevalence significantly increases over time, particularly after the age of 65 it doubles every five years [2].

There is a correlation between ageing, dementia and depression. Depression affects predominantly older people, with its prevalence going up to $9.3 \%$ in over $60 \mathrm{~s}$ [3]. Changes related to age and chronic physical diseases may contribute to modifications in the brain areas involved in the regulation of mood, respectively the frontostriatal circuits, the amyg- 
dala and the hippocampus, predisposing to depression [4]. Moreover, isolation, relocation, caregiving, and other age-related psychosocial stressors are a significant risk factor for depression in later life [5]. Chronic stress can increase cortisol levels, which contribute to depressive disorders and to the atrophy of the hippocampus, also affecting its cognitive functions [6-8].

Literature shows that the association between depression and dementia is complex and bidirectional. Longitudinal studies suggest that recurrent depressive symptoms in adulthood, late-life depression and chronic subsyndromal depression significantly increase the risk of dementia over time [9-11]. Therefore, early-life depression may be considered a risk factor for dementia in older age and late-life depression may be a prodrome to dementia. Likewise, dementia can be considered a risk factor for depression. People with dementia (PWD) have a higher rate of overt depression, ranging from $20 \%$ in AD to 37\% in Frontotemportal Dementia and 50\% in Vascular Dementia [12-14], whilst depressive symptoms have been reported in $30 \%-50 \%$ of AD patients [15].

Depression in dementia can be underdiagnosed because its presentation in older people can differ from the Diagnostic and Statistical Manual of Mental Disorders (DSM)-5 [16] criteria which are mostly based on adult populations, and because the cognitive symptoms may overlap with the dysfunctional signs of dementia [17].

\subsection{Dementia as a social disease: the impact of dementia on informal carers' health and social life}

Dementia affects the person suffering from it as well as all his/her family and social network. In fact, most PWD live at home assisted by their family members (e.g., spouses and children) who become their main carers [18].

Beyond the multiple cognitive impairment, the behavioral and psychological symptoms experienced by PWD, such as depression, personality changes, aggressiveness, delusions, hallucinations, wandering, and sleep disturbances $[19,20]$, are challenging to accept and manage by informal carers. The latter therefore often tend to reduce the occasions on which they can be in public in order to avoid embarrassing situations, this leading them to social isolation defined as the lack of social contact, relationships, and social integration [21-23]. PWD are considered one of the most socially excluded groups of older people [24,25] and so are their informal carers [26], with a negative impact on their health related quality of life [27]. The association between social isolation and dementia is bilateral because the former is both the consequence and one of the driving forces behind the latter. Empirical evidence greatly highlights the association between social isolation and dementia and vice versa [28]. Family carers of PWD subsequently report increased rates of caring strain, physical and mental health problems, and experience difficulties in maintaining employment, leisure activities and family interactions which impacts their quality of life [28]. Moreover, family carers can experience fatigue, frustration, a sense of inadequacy, a feeling of being trapped and loneliness, the latter worsened by social exclusion and stigma characterizing the care recipients. Furthermore, increased caregiver burden in relation to behavioral or depressive symptoms in dementia has been shown to lead to neglect or physical and psychological abuse by the family carer [29,30]. With the advent of the COVID-19 pandemic, older people, including PWD, have been called to shelter because they have been identified at high risk for contracting SARS-CoV-2 infection [31] and the rules around social distancing have restricted access to community services for PWD and their carers. During the outbreak, few care services have adapted to providing remote support to PWD [32] and most Centers for Cognitive Disorders and Dementia, markedly reduced their activities. As consequence, many PWD experienced faster symptom deterioration during the pandemic [33]. Social isolation caused a decline in memory function, increased anxiety and depression symptoms, especially in individuals with mild to moderate dementia, and worsened the general health condition of informal carers who experienced increased tiredness, overwhelming feelings, health problems, sleep disturbances, and greater irritability associated with social isolation [34]. A clear association has been 
found between the cognitive decline of older PWD during the lockdown and the increase of family carers' psychological distress [35].

In light of the above, psychotherapy should involve the person in need as well as the family carer as an individual belonging to his/her environment in order to put in place appropriate measures for treating dementia and correlated depressive symptoms. GT prioritizing the relationship between the person and his/her social contacts and the environment, may be an effective approach for mitigating the disruptive impact of depression on PWD and family carers, as described in the paragraphs below.

\subsection{The contest of dementia diagnosis and formal care: a snapshot of Italy and Mexico}

The care and the management of dementia differs country by country depending on epidemiological figures, the Long-Term Care (LCT) system, welfare regimes, and level of country income. In Latin America the prevalence of diagnosed dementia in 2010 was 3.10 million cases compared to 9.95 million in Europe and an increase of $393 \%$ is expected by 2050 compared to an $87 \%$ rise in Europe [36]. The increasing number of diagnosed PWD will contribute to growing inequalities in low- and middle-income countries where $15 \%$ of dementia-related costs is covered by social care compared to $40 \%$ in high-income countries [37].

Despite Europe being considered a wealthy continent with a better health system compared to Latin America, the quality and level of care provided to PWD from the time of diagnosis onwards can differ significantly in each country.

This article shows an intervention research protocol targeted to PWD and depressive symptoms living in Mexico and in Italy. Since these countries have different LTC systems and gross domestic products (GDP), the epidemiological and socio-economic contexts have been explored and are described in the following sub-paragraphs, in order to provide the rationale of the study protocol presented in this paper.

In Italy, the overall number of PWD will almost double from 2018 to 2050 reaching the figure of $2,247,715$ individuals that will represent $4.13 \%$ of the overall population compared to $2.12 \%$ in 2018 [38,39], thus keeping in line with the European and western world trend. In the country, diagnosis of dementia is frequently made in hospital when the person is at a middle-advanced stage of the illness, contrary to what happens in other European countries such as the United Kingdom where health services considerably promote early diagnosis and Memory Services have a great focus on providing support at the early stages of the disease. Although the attention is to slow down the progress of the disease, in Italy there is not a clear strategy to facilitate early diagnosis nor to promote a personcentred care aimed at keeping the person independent and cutting the cost of LTC. The diagnosis of dementia is often not even communicated to the patients, but only to their family members, and working on acceptance of the new condition can be problematic.

Once received the diagnosis, families often attempt to keep the PWD at home and manage the care assistance by using support both from public and private sources. The National Health Service offers home healthcare services (Assistenza Domiciliare Integrata, ADI) which include specialist assistance by nurses, physiotherapists and medical doctors, and does not include psychotherapy, and only for an average of 16 hours per year per patient [40]. The last available data reported that only 9.5\% of PWD received this type of support in 2016 [41]. At a local level, the municipality partially offers, through a voucher scheme, another type of home social care service (Servizio di Assistenza Domiciliare, SAD) that covers basic individual needs e.g., food intake for highly dependent older people. The provision of SAD can vary significantly across Italian areas and is not well integrated with the ADI [42]. Moreover, it is not considered a sufficient nor appropriate service for covering the needs of PWD and their family carers [43]. Another type of dementia care voucher, that is not distributed evenly across the country, consists of funding to access a psycho-educational intervention in "Alzheimer Day Centers" which are available only to $12.5 \%$ of families despite this being part of an essential level of assistance [41]. Finally, the State Care Allowance (Indennità di Accompagnamento) is a universal and not 
means-tested monetary transfer of 522,10 EUR per month provided to the person in need of care. Such a contribution often partially covers the cost of live-in (migrant) care workers who can guarantee $\mathrm{H} 24$ home assistance to PWD [41]. This means that families cover most of the cost of care with their own budget. [44].

Epidemiological figures in Mexico mirror Latin America's trend with a prediction of nearly 1.5 million cases by 2030 and an increase of $414 \%$ between 2010 and 2050 [45]. Only in recent years has there been a Governmental effort to boost the proposal of the first national Alzheimer's Plan of Action (APA) [46]. APA aims at promoting PWD and their families' well-being by empowering services of the Mexican Healthcare System and cooperating institutions. Guidelines and strategies for implementing specific actions have been published, the second strategy being "To ensure access to quality services" [46,47]. However, currently many families in rural areas and living in poverty do not have access to specialized dementia centers nor to training and guidance for home care assistance. Community-type care has emerged as a response and as an alternative. However, the aim of this type of support is limited at providing information to sensitize the community, and lacks an ethical approach based on empirical research prior to applying the intervention.

\subsection{Psychological support and psychotherapy interventions: Gestalt Therapy view on dementia and on depression}

In light of the above, very little is offered in terms of psychological support for PWD and their families to hinder depression, both in Italy and in Mexico, both when PWD live at home and when in residential or semi-residential institutions. Conversely, the treatment of depression is very important because depression affects the relationship between PWD and their family, as the person becomes more apathetic and less cooperative. As a result, it becomes even more difficult for family members to take care of the person with dementia, with negative effects on the health of the informal carer and on the level of care required, on the social dimensions (i.e. social inclusion and participation), and ultimately on the progression of the disease. In fact, some studies have underlined the association of depression in dementia with decreased quality of life [48] and level of autonomy in performing the Instrumental Activities of Daily Living (IADL) [49], quicker progression of cognitive decline and earlier admission to residential facilities [50] and, as described above a clear association to the interruption of social relationships [27]. In light of this, it is crucial to early identify the symptoms of depression in people with dementia and to offer them appropriate psychotherapeutic support.

Gestalt therapy (GT) is a phenomenological, aesthetic and field-oriented approach based on humanistic and holistic principles. GT theory considers that through human development individuals learn ways of coping and relating to others in their family environment, which learning become then generalized and automatic, inscribed in the procedural memory that tends to be economically fast for our brain and kept out of awareness [51]. The primary aim of GT is to restore the wholeness of the experience of the person, including bodily feelings, movements, emotions, and the ability to creatively adjust to environmental conditions, by empowering the awareness in the present moment. The therapist works to address the implicit knowledge of patients by accessing her/his non-verbal and creative resilience. The phenomenological principle addresses the experience of the client in its wholeness by paying attention to nonverbal cues over the cognitive ones. For instance, rather than focusing on the memory of an event, the emphasis is on developing the ability to stay in the therapeutic relationship harmonically, like in a dance, and learn how to express the own wish for relational contact and sense of agency. The aesthetic principle allows to look at the client's experience through all human senses; instead of using cognitive criteria to evaluate the capacity for relational contact of the client, the therapist can consider other resilient aspects like their body movements, emotions, level of arousal and direction of energy (intentionality for contact) [52]. The field principle allows the therapist to work with reciprocity and consider synchronicity as a cue to evaluate the ability of the client [53]. An effective intervention has to be focused on the relational "dance" that 
therapist and client co-create more than on achieving a certain individual goal. Whilst a goal is agreed at the time of the therapy contract, the focus of the work will not be on the goal, which will only be discussed at review points and at the end of therapy. The "dance of reciprocity" will make the client able to fulfill also cognitive goals, because this relational "dance" will support their ability to cope and adjust to the environment [52]. In this way the intervention will be on the function of the client to flexibly adapt to their environment, in different and creative ways, rather than on acquiring certain behavioral aims.

Because of Gestalt therapy's experiential and experimental nature, there is a lack of empirical research supporting its evidence. A recent systematic review selected eleven scientific papers on the effectiveness of the approach confirming the validity of GT as a clinical model [54]. In favor of the use of GT with PWD, it is possible to consider some neuropsychological and relational aspects of the approach together with the common residual abilities of PWD. First of all, it is important to take into account that although working memory, short-term memory and language can become impaired with the progression of dementia, emotional and procedural memory are retained skills that may decay only at the very late stages of the disease [55]. GT focuses on procedural memory, or nondeclarative memory, that is related to embodied learning on how things are done, through the selection, use, retention and recall of perceptual, cognitive and motor skills [51]. This procedural level should be the priority in the therapeutic process when working with PWD and in general to promote a long-term change. Gestalt therapists work on attunement to movement, facial expressions, posture, tone of voice, the affective content of communication, supporting difficult emotions that emerge in the relational field with the patient [56]. By focusing on the importance of the person's adaptation to the new situation (neurological, emotional and social), gestalt therapists help the patient to create new responses to the changing environment, thus addressing the social dimension of the illness [54], by supporting resources, instead of being concerned with what does not work and the continuous losses. The changing environment for PWD relates not only to the outside world but also to their experience of self in relation to the change and challenges that dementia brings, which adds a level of complexity when working with these patients.

Considering the aims of this study, it is also relevant to define depression in GT, which is viewed from a phenomenological and aesthetic perspective [57]. GT offers an original attempt to reformulate depression in terms of experience of "contact" with the environment. "Contact" in GT is the ability to interact with the environment with the aim to fulfill a need. GT connects depression with 5 dimensions related to the organism/environment field, which have also been observed to co-occur in both the client and the therapist: 1) depression is considered as the lack of connection with the environment; 2) a depressive experience is viewed as the renunciation of the desire to be desired rather than related to the frustration of past desires not being fulfilled; 3) depression is seen as the inability to transcend oneself; 4 ) depression is conceptualized as a lack of hope rather than a lack of happiness; 5) depression indicates the inability to feel oneself - from the bodily sensations level to the emotional one [58].

The effectiveness of GT for depression has been supported by a small number of studies, one of which involved geriatric patients with depression and anxiety [54,59]. In fact, despite the potential effectiveness of this approach, there is still a gap in research on using GT for PWD and for those with depressive symptoms.

The experiential, creative and flexible aspects of GT can provide a unique way of working therapeutically with PWD because of the focus on the non-verbal part of the experience, on the residual abilities of the person, and on the use of body movement and art as aims of communication, which can greatly facilitate the therapeutic engagement towards the attainment of therapeutic goals.

GT can offer a way of working with PWD and depression that can easily incorporate access to other forms of support and activities, not only in a talkative way but in a practical one. The creative flexibility implicit in the approach allows therapist and patient to develop together the therapeutic path which can be extended to the outside world, therefore 
going beyond the walls of the therapy room. For instance, they can agree that as part of therapy they attend a walking group or go for a bus trip together.

\subsection{Proposed research aims}

The study wants to investigate the efficacy of GT for PWD who are affected by depression. Additionally, it wants to observe the indirect effects of the intervention on family carers. Its primary research questions are:

1. Is there any pre-post treatment improvement?

2. Is the change clinically meaningful?

3. Can the improvement be attributed to the therapy process?

4. Can the improvement be also observed in the caregiver?

\section{Materials and Methods}

\subsection{Selection of the research design: Single Case Experimental Design}

Scientific literature offers plenty of evidence that non-pharmacological interventions are the best choice for managing the psychological and behavioral symptoms of dementia, including depression $[60,61]$. Pharmacotherapy for depression in dementia has unclear outcomes and antidepressants may accelerate the progression of dementia [62-64]. Psychological therapies, particularly Cognitive Behavioral Therapy (CBT), are among the most evidenced approaches to treat depression in dementia [65].

In a systematic review Cheston and Ivanecka [66] highlight that there is poor evidence of the efficacy of psychotherapy for the treatment of non-cognitive symptoms in dementia patients. The authors state that manualized interventions such as CBT are best evidenced because the treatment can be measured through the RCT model, yet RCTs have important limitations in psychotherapy research. The American Psychological Association [67] states that the Single-Case time series design can be as valid as RCTs. Moreover, Single Case Experimental Designs (SCEDs) can ensure high-quality research for small heterogeneous groups in clinical settings, allowing the focus on the unique needs of patients [68] rather than on a set of given techniques. It is well known that dementia affects each person differently therefore it is crucial to adopt person-centred approaches in dementia care and to tailor the psychotherapeutic treatment to the needs and presentation of each patient. Gestalt psychotherapy can be a flexible and user-centred approach that, for the reasons mentioned above, could be proved efficient in treating depression in dementia. Considering the qualities of GT introduced above, SCEDs appear to be the best type of investigation to measure the effectiveness of the Gestalt approach.

The A-B-A single case experimental design will be used for the purpose of the study as explained previously.

The research method is divided in three phases: an " $\mathrm{A}$ " baseline period of two weeks between the assessment session " 0 " to the initial psychotherapy session; $a$ " $B$ " intervention period of minimum 8 Gestalt psychotherapy sessions; an " $\mathrm{A}$ " follow-up period of six months after the end of the intervention, with measurements after two weeks and after six months. The baseline phase includes two weeks of daily assessment in which individual target complaints are identified and baseline levels of depression and anxiety are recorded. The target complaints will be completed daily during the intervention phase. The follow-up phase also includes a daily assessment for the first two weeks plus the final administration of outcome measures at week two and at week twenty-four (Figure 1). 

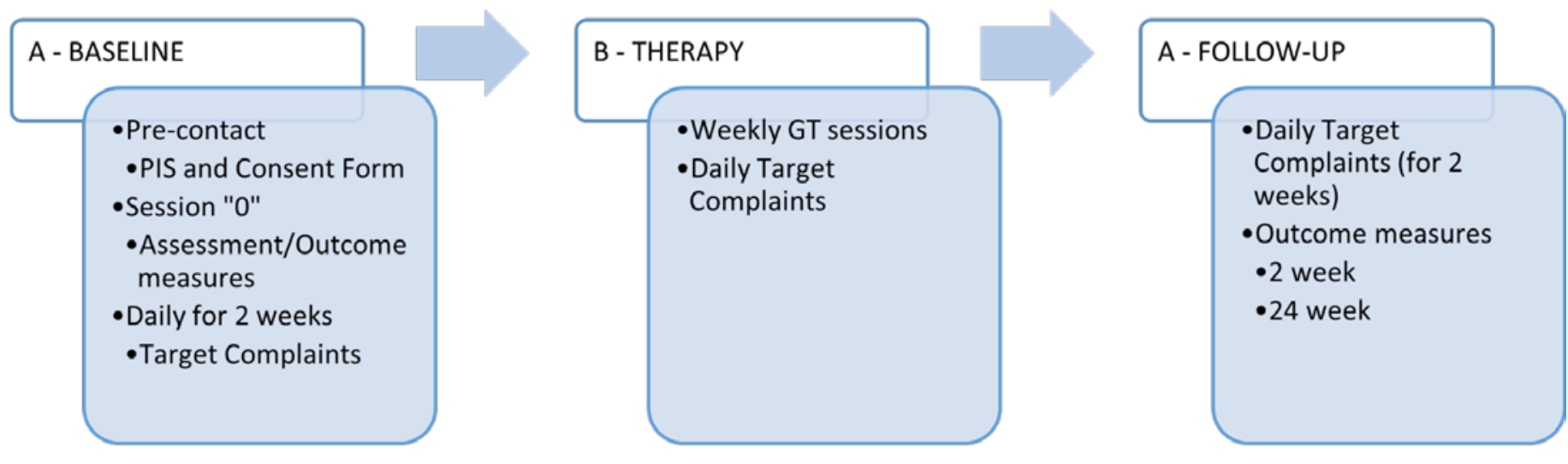

Figure 1. A-B-A design of the study.

\subsection{Study setting and intervention}

The intervention will consist of individual GT sessions, delivered weekly for the duration of one hour. The minimum number of sessions is eight, whilst a maximum number will be recorded depending on individual needs of the participants with dementia.

The intervention will be delivered in psychotherapeutic settings provided by public health clinics or private therapy centers.

Participants will be provided with an information sheet describing the study purpose and procedure and they will be asked to sign a consent form for the data collection and treatment. The full anonymity of the individuals taking part in the treatment will be ensured according to the General Data Protection Regulation (2016/679) [69] and the national laws on the privacy of sensitive and health-related data in force in Italy and in Mexico. The study protocol will be submitted to the attention of the competent ethics committees.

\subsection{Sample and recruitment}

Recruitment will involve two groups of participants: 6 Gestalt psychotherapists and 20 PWD suffering from depression, 10 in Italy and 10 in Mexico respectively.

PWD will be recruited through public health clinics in Italy and Mexico, whilst Gestalt psychotherapists through national professional bodies and organizations.

To be part of the study, PWD will need to: a) provide evidence of formal diagnosis by a medical doctor; $b$ ) having received the diagnosis within the last two years; $c$ ) be able to engage in therapy; d) display symptoms of depression and/or anxiety; e) maintain the psychopharmacological treatment during the investigation; f) live in the community.

Exclusion criteria are: a) diagnosis of dementia at a moderate-severe stage; b) display of challenging behaviors, delusions or psychotic symptoms; c) high level of risk and safeguarding issues; d) parallel therapy/intervention for the same target complaint (e.g. psychoeducational groups); e) variation in psychopharmacological treatment during the investigation; f) admission to hospital/residential care.

Gestalt psychotherapists included will need to: a) agree to participate, be recorded during sessions and fill questionnaires; $b$ ) be accredited Gestalt psychotherapists with previous experience of working with PWD; c) have access to supervision with a Gestalt supervisor.

\subsection{Measures and data collection tools}

Overall, data will be collected by questionnaires and audio/video recording. Outcome measures will gather quantitative data on the symptoms, quality of life, carers' burden and mutuality, and will be repeated at session " 0 ", at the final session, and follow-up 
whilst target complaints will be repeated daily and will be used for the time-series analysis. All the measures and related administration time points are shown in Table 1 and described below.

The Neuropsychiatric Inventory Questionnaire (NPI) was developed by Cummings et al. [70] and validated by Binetti et al. [71] for the Italian population and by Zepeda et al. [72] for the Mexican population. It measures behavioral symptoms related to dementia conditions assessing twelve sub-domains: delusions, hallucinations, agitation/aggression, depression/dysphoria, anxiety, elation/euphoria, apathy/indifference, disinhibition, irritability/lability, motor disturbance, night-time behaviors, appetite/eating. Additionally, a Caregiver Distress Scale was included for measuring the psychological impact of the neuropsychiatric symptoms on family caregivers [73]. The NPI anxiety score will be used to assess the level of anxiety of participants at pre-post treatment. The test has good internal consistency, content validity, and inter-rater/test-retest reliability.

The Clinical Dementia Rating (CDR) is a multidimensional tool that measures the level of cognitive and functional impairment from the patient and proxy point of view. The information is collected by independent semi-structured interviews. Assessed domains include: Memory, Orientation, Judgment and Problem Solving, Community Affairs, Home and Hobbies, and Personal Care. The score ranges from no impairment to severe dementia on a 5 -point scale. A CDR score of $0.5,1$ or 2 would meet the criteria for dementia in the mild to moderate stage. CDR is widely used in research for its well established validity and reliability [74,75].

The Geriatric Depression Scale (GDS) is a screening tool consisting of a 30-item questionnaire for measuring symptoms of depression in older people. It is widely used in research and clinical settings because of its sensitivity (92\%) and specificity (89\%). GDS seems reliable to detect depression in mild to moderate dementia depending on the level of insight of the person, therefore it needs caution in administration [76].

The Target Complaints (TC) Daily Form [77] is a co-constructed self-report measure of 3 central idiosyncratic issues and 1 general wellbeing item. The items are identified together with the therapist at session " 0 ", then rated daily on a Likert-scale from 1 to 10 where 1 equals "not bothered at all" and 10 "could not be worse". Each complaint should be concrete, measurable/quantifiable, frequent, stable without treatment, relatively independent from one another, specific, and negatively formulated. The main instruction that helps identifying and scoring the target complaints is "Please record up to three presenting concerns that brought you to therapy. Circle the number that best represents your personal degree of discomfort as a result of each issue." Improvement on global assessments and target complaints correlated at .71 [78], and test-retest reliability was at .76 [79].

Quality of Life-Alzheimer's Disease (QOL-AD) is a self-report tool for the PWD and their proxy/caregiver based on 13 items of multiple domains including physical health, mood, marriage and fun. QOL-AD has excellent internal consistency and inter-rater reliability, and good criterion and construct validity [80].

The Ad-hoc Common Assessment Tool is a questionnaire aimed at obtaining information on the level of care required by the PWD. It is an idiosyncratic and non-standardized tool containing questions such as the weekly hours of caregiving and the type of formal or informal support involved.

Zarit Burden Inventory (ZBI) measures the level of caregiver burden and is widely used in dementia research for its good validity and internal consistency reliability (Cronbach's alpha coefficient of .92) [81,82]. The revised version contains 22 items, each one endorsed on a 5-point scale ranging from 0 (Never) to 4 (Nearly Always). It provides a total score, and only recently the author identified subdomains and related scores using a concept mapping method. Subdomains are: Burden in the relationship, Emotional wellbeing, Social and family life, Finances, Loss of control over one's life (Scaling and Scoring Version 6.0: July 2018, available at https://eprovide.mapi-trust.org/instruments/zarit-burden-interview). 
The Mutuality Scale [83] is a self-report questionnaire that measures the construct of mutuality as defined by the authors: "the positive quality of the relationship between caregiver and care-receiver" (p. 376). The scale consists of 15 items, divided in subdomains (love, shared pleasurable activities, shared values, and reciprocity) rated on a five-point scale ranging from 0 (not at all) to 4 (a great deal). High scores indicate high mutuality which is associated with decreased caregiver's stress [83-85] and lower caregiver burden [86].

Clinical Outcomes in Routine Evaluation - Outcome Measure (CORE-OM) is a 34item self-report measure of psychological distress comprising 4 domains: wellbeing, symptoms, functioning and risk [87]. CORE-OM is widely used in psychotherapy research and clinical settings for its good reliability and test-retest stability [88]. A study on the older adult population reported that the tool can be used with people with mild cognitive difficulties, and recommended the use of cut-off points identified by the latter investigation due to the risk of false positives [89].

The Gestalt Therapy Fidelity Scale (GTFS) [90] is a 25-item tool assessing the treatment fidelity on a 25-minute video-tape of GT sessions. Video recordings are randomly selected at the end of the first treatment and external assessors evaluate if the treatment is Gestalt or not by scoring each item using a "yes" or "no" rate. In case an intervention falls under the cut-off score of 11 , the therapist will be excluded from the research and the intervention will be considered null for the purposes of this study.

Table 1. Outcome measures with related variables, subjects involved and time of data collection.

\begin{tabular}{|c|c|c|c|}
\hline Instrument & Variable & Point of view & When it is used \\
\hline $\begin{array}{l}\text { Neuropsychiatric } \\
\text { Inventory (NPI) }\end{array}$ & $\begin{array}{l}\text { Neuropsychiatric } \\
\text { symptoms (patient) } \\
\text { and Caregiver } \\
\text { Distress }\end{array}$ & $\begin{array}{l}\text { Expert judge } \\
\text { interviews } \\
\text { caregiver }\end{array}$ & Session “ 0 ” \\
\hline $\begin{array}{l}\text { Clinical Dementia } \\
\text { Rating (CDR) }\end{array}$ & $\begin{array}{c}\text { Cognitive } \\
\text { symptoms and } \\
\text { dementia diagnosis }\end{array}$ & $\begin{array}{c}\text { Expert judge } \\
\text { interviews patient }\end{array}$ & Session " 0 " \\
\hline $\begin{array}{c}\text { Geriatric } \\
\text { Depression Scale } \\
\text { (GDS) }\end{array}$ & $\begin{array}{l}\text { Depressive } \\
\text { symptoms }\end{array}$ & Patient self-report & $\begin{array}{l}\text { Session “0”, final } \\
\text { session and follow } \\
\text { up }\end{array}$ \\
\hline $\begin{array}{l}\text { Anxiety indicated } \\
\text { by an NPI-A score } \\
\text { of } 4 \text { or more }\end{array}$ & Anxiety symptoms & $\begin{array}{l}\text { Expert judge } \\
\text { interviews } \\
\text { Caregiver }\end{array}$ & $\begin{array}{l}\text { Session "0", final } \\
\text { session and follow } \\
\text { up }\end{array}$ \\
\hline $\begin{array}{l}\text { Target Complaints } \\
\text { (TC) }\end{array}$ & $\begin{array}{l}\text { Specific results of } \\
\text { therapy }\end{array}$ & $\begin{array}{l}\text { Patient self-report } \\
\quad \text { (after } \\
\text { co-construction of } \\
\text { instrument with a } \\
\text { therapist) }\end{array}$ & $\begin{array}{c}\text { Co-constructed at } \\
\text { session } \\
\text { "0". } \\
\text { Client completes } \\
\text { questionnaire every } \\
\text { day } \\
\text { after that until } \\
\text { follow up } \\
\text { session }\end{array}$ \\
\hline
\end{tabular}


QOL-AD Quality of Life

Ad-hoc Common

Assessment Tool

\author{
Zarit Burden \\ Inventory
}

The Mutuality

Scale

CORE-OM

Gestalt Therapy

Fidelity Scale (GTFS)
Level of care

Caregiver level of
stress

Mutuality of

relationship

Overall results of therapy

Patient self-report

Treatment fidelity
Expert judge

Expert judge

interviews patient and family

caregiver selfreport

Family member self-report

Family member self-report

Patient and caregiver selfreport observes therapy sessions
Session " 0 ", final session and follow up

\section{Session "0"}

Session " 0 ", final session and follow up

Session " 0 ", final session and follow up

Session " 0 ", final session and follow up

End of first therapy treatment

\subsection{Data Analysis}

SPSS Statistics will be used to generate descriptive statistics about the demographic profiles of participants.

In order to observe if there is any pre-post improvement and its size, three types of analysis will be carried out: a) visual analysis, comparing the target complaint (TC) scores during the three phases; $b) \mathrm{R}$ coefficient (Pearson's correlation) and $\mathrm{p}$ value $(<0.05)$ of TC scores to test if any change happens between the Baseline and Follow-up phases, for which purpose the SMA software will be used [91]; c) Mean Baseline Reduction (MBLR) for obtaining the effect size for single case designs [92-94] as follows: phase.

MBLR $=(\text { Mean of Baseline phase }- \text { Mean of Follow-up phase })^{*} 100 /$ Mean of Baseline

The significance of any observed change ( $\mathrm{p}$ value $<0.05$ ) will be calculated by comparing outcome measures for the symptoms (GDS; NPI-A) and quality of life (QOL-AD) at session " 0 ", final session and follow-up (week two and week twenty-four). Additionally, the significance of indirect effect will be obtained by comparing data collected from family caregivers (QOL-AD family version; Caregiver Burden Scale; The Mutuality Scale) at the phases aforementioned.

In case an improvement is observed, its correlation to the therapy process will be calculated by the R-squared value ( $p$ value of the F-test of the overall regression significance).

Blind raters will assess the fidelity of the approach by rating a small sample of sessions through the GTFS.

\section{Discussion}

Depression in dementia can be treated with the use of psychosocial interventions, psychotherapy being part of this category. Any therapeutic approach that is used to work with PWD should be flexible, person-centred and creative in order to meet the needs of 
the person, as it has been well underlined by Kitwood and followers $[95,96]$. These qualities are already central in GT and testify to the suitability of the approach for working with this population.

The design of this study mainly followed indications for the development of a SCED in GT as recommended by GT authors with experience in research $[97,98]$. The aim of observing the effectiveness of the approach has been enriched by considering the context of the experience of living with dementia, which inevitably includes informal carers, who are often family members. Therefore, the benefits of receiving GT as treatment should be observed also from the perspective of the main family carer. This added aspect is in line with the GT field principle, i.e., that the embodied learning from the therapeutic "dance" can elicit relational adaptations (a new dance) in the family system.

Providing psychotherapeutic support from the earliest stages of dementia allows the patients to cope with the disease from the very beginning, integrating it into their lives and also becoming protagonists of the future therapeutic choices. A grieving reaction, accompanied by an emotional crisis, which may include depression, may be experienced from the moment of diagnosis [99], as a response to it, or even before diagnosis [100], as the person's life begins to be affected by the initial symptoms of dementia. The support received through a psychotherapeutic intervention can help the person to cope with losses in order to create continuous new adaptations. GT's holistic approach makes it possible to undertake a pathway to support the physiological, emotional and cognitive ground of the patient that may be disrupted by the dementia condition.

It is expected that the results obtained from this research can submit significant data to support the impact between pre-post improvement, as well as whether the observed changes are clinically meaningful. It will be possible to review the attribution of these changes to the Gestalt therapeutic work.

An indirect effect on the family carers may be observed as a result of the researched intervention, which may manifest in a reduction of their stress levels or emotional burden, and consequent improvement of the relationship with those they care for.

The statistical comparison strengthens the present evidence-based study on the efficacy of GT with people in these circumstances, and also opens a whole field of work and possibilities for undertaking future studies.

\section{Limitations and challenges of the research protocol}

The project was developed prior to the start of the COVID-19 pandemic and only considered an in-person setting for face-to-face therapy. Since we do not have sight of when the pandemic will terminate and considering that this time could come in several years, the inclusion of only in-person settings can be an important limitation. On the other side, an online setting may bring restrictions to some valuable aspects of GT particularly when applied to PWD if we consider the cognitive difficulties of patients that already imply some limits. Additionally, people who live in Italy and Mexico tend to receive a diagnosis at their mid if not late stages of the condition which means that they will experience further limitations in using and engaging in tele-therapy.

In relation to data collection, qualitative data may add value to quantitative figures. At this stage, the protocol only includes a quantitative methodology that can guarantee rigor and reproducibility. As the therapy sessions will be video-recorded, there might be a possibility of further analysis of the content of the meetings (with the consent of the participants) as part of a secondary research project.

The application of the protocol might encounter some challenges. First of all, there are not many services for PWD that offer psychotherapy, therefore there is not a large number of professionals with experience in the field of dementia. Additionally, the Gestalt psychotherapy community is not as large as that of other approaches such as psychoanalysis, and cognitive-behavioral therapies, so it may be even more difficult to find Gestalt psychotherapists with expertise in dementia. Another challenge that may have an impact on recruitment, may be the stigma of having psychological problems (particularly present 
in the current cohort of older people) as this adds to the stigmatic images related to the dementia condition, and together may hinder acceptance of the need for help. As a final point, the lack of public healthcare provision for PWD means that there is a small availability of free-of-charge psychotherapy treatments in both countries. Therefore, the application of this protocol will depend on the obtainment of a grant and/or the in-kind work of the professionals involved.

\section{Conclusions}

The objective of the study is to observe the potential of GT as an effective intervention for working with PWD and depressive symptoms. The scientific soundness of the SCED methodology will rigorously produce reliable results that, we believe, will confirm the validity of GT with the considered population. Detailed reporting of the protocol can support the replicability of the research in other countries and other settings.

Author Contributions: Conceptualization, A.M., R.B. and F.A.Z.; methodology, A.M., R.B. and F.A.Z.; writing-original draft preparation, A.M., R.B., F.A.Z., M.S-L. and S.S.; writing-review and editing, A.M. and S.S.; project administration, A.M. All authors have read and agreed to the published version of the manuscript.

Funding: The development of this protocol was supported by Ricerca Corrente funding from the Italian Ministry of Health to IRCCS INRCA.

Institutional Review Board Statement: The study will be conducted according to the guidelines of the Declaration of Helsinki, and will be approved by the competent Ethics Committee.

Informed Consent Statement: Informed consent will be obtained from all subjects involved in the study.

Acknowledgments: We want to thank in advance all the people living with dementia and their family members who will participate and support the participation in the research. A special thank you goes to Pablo Herrera Salinas for his great help with the development of the research design.

Conflicts of Interest: The authors declare no conflict of interest.

\section{References}

1. World Health Organization. Global status report on the public health response to dementia; WHO: Geneva, Switzerland, 2021; pp. 25.

2. Hugo, J.; Ganguli, M. Dementia and cognitive impairment: epidemiology, diagnosis, and treatment. Clin Geriatr Med. 2014, 30, 421-442; DOI: 10.1016/j.cger.2014.04.001.

3. Sjöberg, L.; Karlsson, B.; Atti, A.R.; Skoog, I.; Fratiglioni, L.; Wang, H.X. Prevalence of depression: Comparisons of different depression definitions in population-based samples of older adults. J Affect Disord. 2017, 15, 123-131; DOI: 10.1016/j.jad.2017.06.011.

4. Sexton, C.E.; Mackay, C.E.; Ebmeier, K.P. A Systematic Review and Meta-Analysis of Magnetic Resonance Imaging Studies in Late-Life Depression, Am J Geriatr Psychiatry 2013, 21, 184-195; DOI: 10.1016/j.jagp.2012.10.019.

5. Alexopoulos, G.S. Depression in the elderly. Lancet 2005, 365, 1961-1970; DOI: 10.1016/S0140-6736(05)66665-2.

6. Butters, M.A.; Young, J.B.; Lopez, O.; Aizenstein, H.J.; Mulsant, B.H.; Reynolds, C.F. 3rd; DeKosky, S.T.; Becker, J.T. Pathways linking late-life depression to persistent cognitive impairment and dementia. Dialogues Clin Neurosci. 2008, 10, 345-357; DOI: 10.31887/DCNS.2008.10.3/mabutters.

7. Sierksma, A.S.; van den Hove, D.L.; Steinbusch, H.W.; Prickaerts, J. Major depression, cognitive dysfunction and Alzheimer's disease: is there a link? Eur J Pharmacol. 2010, 626, 72-82; DOI: 10.1016/j.ejphar.2009.10.021.

8. Wolkowitz, O.M.; Epel, E.S.; Reus, V.I.; Mellon, S.H. Depression gets old fast: do stress and depression accelerate cell aging? Depress Anxiety 2010, 27, 327-38; DOI: 10.1002/da.20686.

9. Dotson, V.M.; Beydoun, M.A.; Zonderman, A.B., Recurrent depressive symptoms and the incidence of dementia and mild cognitive impairment. Neurology 2010, 75, 27-34; DOI: 10.1212/WNL.0b013e3181e62124.

10. Borza, T.; Engedal, K.; Bergh, S.; Selbæk, G. Older people with depression - a three-year follow-up. Tidsskr Nor Laegeforen 2019, 139; DOI: 10.4045/tidsskr.18.0968.

11. Oh, D.J.; Han, J.W.; Bae, J.B.; Kim, T.H.; Kwak, K.P.; Kim, B.J.; Kim, S.G.; Kim, J.L.; Moon, S.W.; Park, J.H.; Ryu, S.H.; Youn, J.C.; Lee, D.Y.; Lee, D.W.; Lee, S.B.; Lee, J.J.; Jhoo, J.H.; Kim, K.W. Chronic subsyndromal depression and risk of dementia in older adults. Aust N Z J Psychiatry 2021, 55, 809-816; DOI: 10.1177/0004867420972763. 
12. Kuring, J.K.; Mathias, J.L.; Ward, L. Prevalence of Depression, Anxiety and PTSD in People with Dementia: a Systematic Review and Meta-Analysis. Neuropsychol Rev. 2018, 28, 393-416; DOI: 10.1007/s11065-018-9396-2._.

13. Park, J.H.; Lee, S.B.; Lee, T.J.; Lee, D.Y.; Jhoo, J.H; Youn, J.C.; Choo, I.H.; Choi, E.A.; Jeong, J.W.; Choe, J.Y.; Woo, J.I.; Kim, K.W. Depression in vascular dementia is quantitatively and qualitatively different from depression in Alzheimer's disease. Dement Geriatr Cogn Disord. 2007, 23, 67-73; DOI: 10.1159/000097039.

14. Ballard, C.; Neill, D.; O'Brien, J.; McKeith, I.G.; Ince, P.; Perry, R. Anxiety, depression and psychosis in vascular dementia: prevalence and associations. J Affect Disord. 2000, 59, 97-106; DOI: 10.1016/s0165-0327(99)00057-9.

15. Zubenko, G.S.; Zubenko, W.N.; McPherson, S.; Spoor, E.; Marin, D.B.; Farlow, M.R.; Smith, G.E.; Geda, Y.E.; Cummings, J.L.; Petersen, R.C.; Sunderland, T. A collaborative study of the emergence and clinical features of the major depressive syndrome of Alzheimer's disease. Am J Psychiatry 2003, 160, 857-66; DOI: 10.1176/appi.ajp.160.5.857.

16. American Psychiatric Association. Diagnostic and statistical manual of mental disorders, fifth edition: DSM-5. American Psychiatric Publishing: Washington, DC, 2013.

17. Burke, A.D.; Goldfarb, D.; Bollam, P.; Khokher, S. Diagnosing and Treating Depression in Patients with Alzheimer's Disease. Neurol Ther. 2019, 8, 325-350; DOI: 10.1007/s40120-019-00148-5._

18. Harrison, K.L.; Ritchie, C.S.; Patel, K.; Hunt, L.J.; Covinsky, K.E.; Yaffe, K.; Smith, A.K. Care Settings and Clinical Characteristics of Older Adults with Moderately Severe Dementia. J Am Geriatr Soc. 2019, 67, 1907-1912. DOI: 10.1111/jgs.16054.

19. Aalten, P.; Jolles, J.; de Vugt, M.E.; Verhey, F.R. The influence of neuropsychological functioning on neuropsychiatric problems in dementia. J Neuropsychiatry Clin Neurosci. 2007, 19, 50-56; DOI: 10.1176/jnp.2007.19.1.50.

20. Dourado, M.C.; Laks, J. Psychological interventions for neuropsychiatric disturbances in mild and moderate Alzheimer's disease: current evidences and future directions. Curr Alzheimer Res. 2016, 13, 1100-1101; DOI: $10.2174 / 1567205013666160728143123$.

21. Coyle, C.E.; Dugan, E. Social isolation, loneliness and health among older adults. J Aging Health 2012, $24,1346-1363$. DOI:10.1177/0898264312460275.

22. Evans, I.E.M.; Martyr, A.; Collins, R.; Brayne, C.; Clare, L. Social isolation and cognitive function in later life: A systematic review and meta-analysis. J Alzheimer's Dis. 2018, 70, S119-S144. DOI:10.3233/jad-180501.

23. Kuiper, J.S.; Zuidersma, M.; Oude Voshaar, R.C.; Zuidema, S.U.; van den Heuvel, E.R.; Stolk, R.P.; Smidt, N. Social relationships and risk of dementia: A systematic review and meta-analysis of longitudinal cohort studies. Ageing Res Rev. 2015, $22,39-57$. DOI:10.1016/j.arr.2015.04.006.

24. Alzheimer's Disease International. Overcoming the stigma of dementia. Available online: https://www.alz.co.uk/sites/default/files/pdfs/world-report-2012-summary-sheet.pdf (accessed on 01 September 2021).

25. Werner, P.; Mittleman, M.S.; Goldstein, D.; Heinik, J. Family stigma and caregiver burden in Alzheimer's disease. Gerontologist 2012, 52, 89-97; DOI: 10.1093/geront/gnr117.

26. Carers UK. Carers Manifesto. Available online: http://www.carersuk.org/for-professionals/policy/policy-library/carers-manifesto (Accessed on 15 November 2017).

27. Bleijlevens, M.H.; Stolt, M.; Stephan, A.; Zabalegui, A.; Saks, K.; Sutcliffe, C.; Lethin, C.; Soto, M.E.; Zwakhalen, S.M.; RightTimePlaceCare Consortium. Changes in caregiver burden and health-related quality of life of informal caregivers of older people with Dementia: evidence from the European RightTimePlaceCare prospective cohort study. J Adv Nurs. 2015, 71, 1378-91. DOI: 10.1111/jan.12561.

28. Wilks, S.E.; Croom, B. Perceived stress and resilience in Alzheimer's disease caregivers: testing moderation and mediation models of social support. Aging Ment Health 2008, 12, 357-365; DOI: 10.1080/13607860801933323.

29. Mouton, C.P.; Haas, A.; Karmarkar, A.; Kuo, Y.F.; Ottenbacher, K. Elder abuse and mistreatment: results from medicare claims data. J Elder Abuse Negl. 2019, 31, 263-280; DOI: 10.1080/08946566.2019.1678544.

30. Max, W.; Webber, P.; Fox, P. Alzheimer's Disease: The Unpaid Burden of Caring. J. Aging Health 1995, 7, 179-199; DOI:10.1177/089826439500700202.

31. Numbers, K.; Brodaty, H. The effects of the COVID-19 pandemic on people with dementia. Nat Rev Neurol. 2021, 17, 69-70; DOI: 10.1038/s41582-020-00450-z.

32. Giebel, C.; Hanna, K.; Callaghan, S.; Cannon, J.; Butchard, S.; Shenton, J.; Komuravelli, A.; Limbert, S.; Tetlow, H.; Rogers, C.; Eley, R.; Rajagopal, M.; Ward, K.; Gabbay, M. Navigating the new normal: accessing community and institutionalised care for dementia during COVID-19. Aging Ment Health 2021, 28, 1-6; DOI: 10.1080/13607863.2021.1914545.

33. Canevelli, M.; Valletta, M.; Toccaceli Blasi, M.; Remoli, G.; Sarti, G.; Nuti, F.; Sciancalepore, F.; Ruberti, E.; Cesari, M.; Bruno, G. Facing Dementia During the COVID-19 Outbreak. J Am Geriatr Soc. 2020, 68, 1673-1676; DOI: 10.1111/jgs.16644.

34. Azevedo, L.V.D.S.; Calandri, I.L.; Slachevsky, A.; Graviotto, H.G.; Vieira, M.C.S.; Andrade, C.B.; Rossetti, A.P.; Generoso, A.B.; Carmona, K.C.; Pinto, L.A.C.; Sorbara, M.; Pinto, A.; Guajardo, T.; Olavarria, L.; Thumala, D.; Crivelli, L.; Vivas, L.; Allegri, R.F.; Barbosa, M.T.; Serrano, C.M.; Miranda-Castillo, C.; Caramelli, P. Impact of Social Isolation on People with Dementia and Their Family Caregivers. J Alzheimers Dis. 2021, 81, 607-617; DOI: 10.3233/JAD-201580.

35. Borelli, W.V.; Augustin, M.C.; de Oliveira, P.B.F.; Reggiani, L.C.; Bandeira-de-Mello, R.G.; Schumacher-Schuh, A.F.; Chaves, M.L.F.; Castilhos, R.M. Neuropsychiatric Symptoms in Patients with Dementia Associated with Increased Psychological Distress in Caregivers During the COVID-19 Pandemic. J Alzheimers Dis. 2021, 80, 1705-1712. DOI: 10.3233/JAD-201513. 
36. Llibre, Jde.J.; López, A.M.; Valhuerdi, A.; Guerra, M.; Llibre-Guerra, J.J.; Sánchez, Y.Y.; Bosch, R.; Zayas, T.; Moreno, C. Frailty, dependency and mortality predictors in a cohort of Cuban older adults, 2003-2011. MEDICC Rev. 2014, 16, 24-30; DOI: 10.37757/MR2014.V16.N1.6.

37. World Health Organization. Global action plan on the public health response to dementia 2017-2025. WHO: Geneva, Switzerland, 2017; pp. 2-3.

38. Alzheimer Europe. Dementia in Europe Yearbook 2019. Estimating the prevalence of dementia in Europe. Alzheimer Europe: Luxemburg, 2019; pp. 54-55.

39. GBD 2016 Dementia Collaborators. Global, regional, and national burden of Alzheimer's disease and other dementias, 19902016: a systematic analysis for the Global Burden of Disease Study 2016. Lancet Neurol. 2019, 18, 88-106. DOI: 10.1016/S14744422(18)30403-4.

40. Berloto, S.; Notarnicola, E.; Perobelli, E.; Rotolo, A. Italy and the COVID-19 long-term care situation. Country report in LTCcovid.org. International Long Term Care Policy Network, CPEC-LSE, 2020.

41. Italian Alzheimer's Disease Association and CENSIS Foundation (Associazione Italiana Malattia di Alzheimer and Fondanzione CENSIS). L'impatto Economico E Sociale Della Malattia Di Alzheimer: Rifare Il Punto Dopo 16 Anni; Fondazione CENSIS: Rome, Italy, 2016; p. 16.

42. Barbabella, F.; Poli, A.; Chiatti, C.; Pelliccia, L.; Pesaresi, F. The compass of NNA: The state of the art based on data. In Care of Non Self-Sufficient Older People in Italy, 6th Report, 2017-2018; NNA Network Non Autosufficienza, Ed.; Maggioli, S.p.A.: Sant'Arcangelo di Romagna, Italy, 2017; pp. 33-54.

43. Tidoli, R. Domicialirity. In Care of Non Self-Sufficient Older People in Italy. 6th Report, 2017-2018; NNA Network Non Autosufficienza, Ed.; Maggioli, S.p.A.: Sant'Arcangelo di Romagna, Italy, 2017; pp. 77-100.

44. Costa, G. Private Assistants in the Italian Care System: Facts and Policies. Obs. Soc. Br. 2013, 14, 99-117.

45. Alzheimer's Disease International and Bupa. La demencia en América: El coste y la prevalencia del Alzheimer y otros tipos de demencia; ADI: London, UK, 2013, pp. 11-12.

46. Gutiérrez-Robledo, L.M.; Arrieta-Cruz, I. (Eds). Plan de acción Alzheimer y otras demencias. México. 2014, 1st ed.; Instituto Nacional de Geriatría/Secretaría de Salud: México, 2014; pp. 49-62.

47. Gutiérrez-Robledo, L.M.; Arrieta-Cruz, I. Demencias en México: la necesidad de un Plan de Acción [Dementia in Mexico: The need for a National Alzheimer's Plan]. Gac Med Mex. 2015, 151, 667-73.

48. Netuveli, G.; Blane, D. Quality of life in older ages. Br Med Bull. 2008, 85, 113-126; DOI: 10.1093/bmb/ldn003.

49. Lam, L.C.; Tam, C.W.; Chiu, H.F.; Lui, V.W. Depression and apathy affect functioning in community active subjects with questionable dementia and mild Alzheimer's disease. Int J Geriatr Psychiatry 2007, 22, 431-437; DOI: 10.1002/gps.1694.

50. Starkstein, S.E.; Mizrahi, R. Depression in Alzheimer's disease. Expert Rev Neurother. 2006, 6, 887-895; DOI: 10.1586/14737175.6.6.887.

51. Tønnesvang, J.; Sommer, U.; Hammink, J.; Sonne, M. Gestalt therapy and cognitive therapy--contrasts or complementarities? Psychotherapy (Chic). 2010, 47, 586-602; DOI: 10.1037/a0021185.

52. Spagnuolo Lobb, M. The now-for-next in psychotherapy: Gestalt therapy recounted in post-modern society. Istituto di Gestalt HCC Italy Publ. Co.: Siracusa, Italy, 2013

53. Spagnuolo Lobb, M. The Paradigm of Reciprocity: How to Radically Respect Spontaneity in Clinical Practice. Gestalt Rev. 2019, 23, 232-254.

54. Raffagnino, R. Gestalt Therapy Effectiveness: A Systematic Review of Empirical Evidence. Open J. Soc. Sci. 2019, 7, 66-83; DOI:10.4236/jss.2019.76005.

55. Fuchs, T. Embodiment and personal identity in dementia. Med Health Care Philos. 2020, 23, 665-676; DOI: 10.1007/s11019-02009973-0.

56. Meulmeester, F. Risk of Psychopathology in Old Age. In Gestalt Therapy in Clinical Practice: From Psychopathology to the Aesthetic of Contact; Francesetti, G., Gecele, M., Roubal, J., Eds.; Franco Angeli: Milan, Italy, 2013; pp. 281-294.

57. Spagnuolo Lobb, M.; Wheeler, G. Fundamentals and development of Gestalt Therapy in the contemporary context. Gestalt Rev. 2015, 19, 1-24.

58. Spagnuolo Lobb, M. (Istituto HCC Italy, Siracusa, Italy); Garrety, A. (Gestalt Australia \& New Zealand, Fairfield, Victoria, Australia); Iacono Isidoro, S. (Istituto HCC Italy, Siracusa, Italy). Gestalt Perspective on Depressive Experience: a questionnaire on some phenomenological and aesthetic dimensions. Istituto di Gestalt HCC Italy, 2021 (Research Protocol).

59. Drăghici, R. Experiential Psychotherapy in Geriatric Groups. Procedia Soc Behav Sci. 2011, 33, 979-983; DOI:10.1016/j.sbspro.2012.01.268.

60. Azermai, M.; Petrovic, M.; Elseviers, M.M.; Bourgeois, J.; Van Bortel, L.M.; Vander Stichele, R.H. Systematic appraisal of dementia guidelines for the management of behavioural and psychological symptoms. Ageing Res. Rev. 2012, 11, 78-86; DOI: 10.1016/j.arr.2011.07.002.

61. de Oliveira, A.M.; Radanovic, M.; de Mello, P.C.; Buchain, P.C.; Vizzotto, A.D.; Celestino, D.L.; Stella, F.; Piersol, C.V.; Forlenza, O.V. Nonpharmacological Interventions to Reduce Behavioral and Psychological Symptoms of Dementia: A Systematic Review. Biomed Res Int. 2015, 218980; DOI: 10.1155/2015/218980.

62. Leyhe, T.; Reynolds, C.F.; Melcher, T.; Linnemann, C.; Klöppel, S.; Blennow, K.; Zetterberg, H.; Dubois, B.; Lista, S.; Hampel, H. A common challenge in older adults: Classification, overlap, and therapy of depression and dementia. Alzheimers. Dement. 2017, 13, 59-71; DOI: 10.1016/j.jalz.2016.08.007. 
63. Moraros, J.; Nwankwo, C.; Patten, S.B.; Mousseau, D.D. The association of antidepressant drug usage with cognitive impairment or dementia, including Alzheimer disease: A systematic review and meta-analysis. Depress Anxiety. 2017, 34, 217- 226; DOI: 10.1002/da.22584.

64. Leong, C. Antidepressants for depression in patients with dementia: a review of the literature. Consult Pharm. 2014, 29, 254-263; DOI: 10.4140/TCP.n.2014.254.

65. Orgeta, V.; Qazi, A.; Spector, A.; Orrell, M. Psychological treatments for depression and anxiety in dementia and mild cognitive impairment: Systematic review and meta-analysis. Br J Psychiatry, 2015, 207, 293-298; DOI: 10.1192/bjp.bp.114.148130.

66. Cheston, R.; Ivanecka, A. Individual and group psychotherapy with people diagnosed with dementia: a systematic review of the literature. Int J Geriatr Psychiatry, 2017, 32, 3- 31; DOI: 10.1002/gps.4529.

67. American Psychological Association. Evidence-based practice in psychology. American Psychologist 2006, 61, 271-285; DOI: 10.1037/0003-066X.61.4.271.

68. Krasny-Pacini, A.; Evans, J. Single-case experimental designs to assess intervention effectiveness in rehabilitation: A practical guide. Ann Phys Rehabil Med. 2018, 61, 164-179; DOI: 10.1016/j.rehab.2017.12.002.

69. Regulation (EU) 2016/679 of the European Parliament and of the Council of 27 April 2016 on the protection of natural persons with regard to the processing of personal data and on the free movement of such data, and repealing Directive 95/46/EC (General Data Protection Regulation).

70. Cummings, J.L.; Mega, M.; Gray, K.; Rosenberg-Thompson, S.; Carusi, D.A.; Gornbein, J. The Neuropsychiatric Inventory: Comprehensive assessment of psychopathology in dementia. Neurology 1994, 44, 2308; DOI: 10.1212/wnl.44.12.2308.

71. Binetti, G.; Mega, M.S.; Magni, E.; Padovani, A.; Rozzini, L.; Bianchetti, A.; Cummings, J.; Trabucchi, M. Behavioral disorders in Alzheimer's Disease: a transcultural perspective. Arch Neurol 1998, 55, 539-544; DOI: 10.1001/archneur.55.4.539.

72. Zepeda, M.U.P.; Guerrero, J.A.R.; Carrasco, O.R.; Robledo, L.M.G. P3-038: Validation of the neuropsychiatric inventory questionnaire in a group of Mexican patients with dementia. Alzheimers Dement. 2008, 4, T527-T528.

73. Kaufer, D.I.; Cummings, J.L.; Christine, D.; Bray, T.; Castellon, S.; Masterman, D.; MacMillan, A.; Ketchel, P.; DeKosky, S.T. Assessing the impact of neuropsychiatric symptoms in Alzheimer's disease: the Neuropsychiatric Inventory Caregiver Distress Scale. J Am Geriatr Soc. 1998, 46, 210-5; DOI: 10.1111/j.1532-5415.1998.tb02542.x.

74. Hughes, C.P.; Berg, L.; Danziger, W.L.; Coben, L.A.; Martin, R.L. A new clinical scale for the staging of dementia. Br J Psychiatry . 1982, 140, 566-572; DOI: 10.1192/bjp.140.6.566.

75. Morris, J.C. The Clinical Dementia Rating (CDR): current version and scoring rules. Neurology. 1993, 43, 2412-4; DOI: 10.1212/wnl.43.11.2412-a.

76. Kørner, A.; Lauritzen, L.; Abelskov, K.; Gulmann, N.; Marie Brodersen, A.; Wedervang-Jensen, T.; Marie Kjeldgaard, K. The Geriatric Depression Scale and the Cornell Scale for Depression in Dementia. A validity study. Nord J Psychiatry. 2006, 60, 3604; DOI: 10.1080/08039480600937066.

77. Battle, C.C.; Imber, S.D.; Hoehn-Saric, R.; Nash, E.R.; Frank, J.D. Target complaints as criteria of improvement. Am J Psychother. 1966, 20, 184-92; DOI: 10.1176/appi.psychotherapy.1966.20.1.184.

78. Shorer, C. Improvement with and without psychotherapy. Diseases of the Nervous System 1970, 31,155 - 160.

79. Frey, J.; Heckel, R.V.; Salzberg, H.C.; Wackwitz, J. Demographic variables as predictors of outcome in psychotherapy with children. J Clin Psychol. 1976, 32, 713 - 721.

80. Logsdon, R.G.; Gibbons, L.E.; McCurry, S.M.; Teri, L. Quality of life in Alzheimer's disease: patient and caregiver reports. J Ment Health Aging 1999, 5, 21-32.

81. Zarit, S.H.; Reever, K.E.; Back-Peterson, J. Relatives of the impaired elderly: correlates of feelings of burden. Gerontologist 1980, 20, 649-655; DOI: 10.1093/geront/20.6.649.

82. Hérbert, R.; Bravo, G.; Préville, M. Reliability, validity, and reference values of the Zarit Burden Interview for assessing informal caregivers of community-dwelling older persons with dementia. Can J Aging 2000, 19, 494-507; DOI:10.1017/S0714980800012484.

83. Archbold, P.G.; Stewart, B.J.; Greenlick, M.R.; Harvath, T. Mutuality and preparedness as predictors of caregiver role strain. Res Nurs Health 1990, 13, 375-384; DOI: 10.1002/nur.4770130605.

84. Godwin, K.M.; Swank, P.R.; Vaeth, P.; Ostwald, S.K. The longitudinal and dyadic effects of mutuality on perceived stress for stroke survivors and their spousal caregivers. Aging Ment Health 2013, 17, 423-431; DOI: 10.1080/13607863.2012.756457.

85. Lyons, K.S.; Stewart, B.J.; Archbold, P.G.; Carter, J.H. Optimism, pessimism, mutuality, and gender: Predicting 10-year role strain in Parkinson's disease spouses. Gerontologist 2009, 49, 378-387; DOI: 10.1093/geront/gnp046.

86. Halm, M.A.; Treat-Jacobson, D.; Lindquist, R.; Savik, K. Caregiver burden and outcomes of caregiving of spouses of patients who undergo coronary artery bypass graft surgery. Heart Lung 2007, 36, 170-187; DOI: 10.1016/j.hrtlng.2006.08.003.

87. Froyd, J.E.; Lambert, M.J.; Froyd, J.D. A review of practices of psychotherapy outcome measurement. J Ment Health 1996, 5, 1115; DOI: 10.1080/09638239650037144.

88. Evans, C.; Connell, J.; Barkham, M.; Margison, F.; McGrath, G.; Mellor-Clark, J.; Audin, K. Towards a standardised brief outcome measure: Psychometric properties and utility of the CORE-OM. Br J Psychiatry 2002, 180, 51-60; DOI: 10.1192/bjp.180.1.51.

89. Barkham, M.; Culverwell, A.; Spindler, K.; Twigg, E. The CORE-OM in an older adult population: psychometric status, acceptability, and feasibility. Aging Ment Health 2005, 9, 235-45; DOI: 10.1080/13607860500090052.

90. Fogarty, M.; Bhar, S.; Theiler, S. Development and validation of the Gestalt Therapy Fidelity Scale. Psychother Res. 2020, 30, 495509; DOI:10.1080/10503307.2019.1571688. 
91. Borckardt, J.J.; Nash, M.R.; Murphy, M.D.; Moore, M.; Shaw, D.; O'Neil, P. Clinical practice as natural laboratory for psychotherapy research: a guide to case-based time-series analysis. Am Psychol. 2008, 63, 77-95; DOI: 10.1037/0003-066X.63.2.77.

92. Campbell, J.M. Efficacy of behavioral interventions for reducing problem behavior in persons with autism: A quantitative synthesis of single-subject research. Res Dev Disabil 2003, 24, 120- 138; DOI: 10.1016/s0891-4222(03)00014-3.

93. Olive, M.L.; Smith, B.W. Effect size calculations and single subject designs. Educ. Psychol. 2005, 25, 313-324; DOI: 10.1080/0144341042000301238.

94. Delfs, C.H.; Campbell, J.M. A quantitative synthesis of developmental disability research: The impact of functional assessment methodology on treatment effectiveness. Behav Anal Today 2010, 11, 4-19; DOI: 10.1037/h0100685.

95. Kitwood, T. The experience of dementia. Aging Ment Health, 1997, 1, 13-22; DOI: 10.1080/13607869757344.

96. Dewing, J. Personhood and dementia: revisiting Tom Kitwood's ideas. Int J Older People Nurs. 2008, 3, 3-13; DOI: 10.1111/j.17483743.2007.00103.x.

97. Herrera, P.; Brownell, P.; Roubal, J.; Mstibovskyi, I.; Glänzer, O. Progetto gestaltico di collaborazione internazionale: Il Caso singolo, progetto di ricerca con serie temporali. Manuale per ricercatori, La Rosa, R.; Tosi, S., Eds.; Istituto di Gestalt HCC Italy: Siracusa, Italy, 2020; pp. 1-24.

98. Herrera, P.; Mstibovskyi, I.; Roubal, J.; Brownell, P. The Single-Case, Time-Series Study. Int J Psychother. 2020, 24, 53-65; 20; DOI: 10.36075/IJP.2020.24.3.6/Herrera/Mstibovskyi/Roubal/Brownell

99. Aminzadeh, F.; Byszewski, A.; Molnar, F.J.; Eisner, M. Emotional impact of dementia diagnosis: exploring persons with dementia and caregivers' perspectives. Aging Ment Health. 2007, 11, 281-90; DOI: 10.1080/13607860600963695.

100. Bopp-Kistler, I. Diagnoseeröffnung und Begleitung [Disclosing the diagnosis and guidance]. Ther Umsch. 2015, 72, 225-31; DOI: $10.1024 / 0040-5930 / a 000669$. 